\title{
Excited State Dynamics of Perylenediimide films with Isopropyl phenyl- and Undecane-Substitution
}

Qiushi $\mathrm{Ma}^{1 *}$, Cheng-Wei $\mathrm{Ju}^{2 *}$, Ruihua $\mathrm{Pu}^{3}$, Wenjie Zhang ${ }^{4}$, Xian $\mathrm{Lin}^{4}$, Yihan Chen ${ }^{1}$, and Weimin $\mathrm{Liu}^{3}$

${ }^{1}$ School of Resource and Environmental Engineering, Hefei University of Technology, Hefei 230009, Anhui Province, China

${ }^{2}$ College of Chemistry, Nankai University, Tianjin 300071, China

${ }^{3}$ School of Physical Science and Technology, ShanghaiTech University, Shanghai 201210, China

${ }^{4}$ Department of Physics, Shanghai University, Shanghai 200444, China

*These authors contributed equally

*Email address:

yhchen@hfut.edu.cn (Y. Chen), liuwm@shanghaitech.edu.cn (W. Liu). 


\begin{abstract}
The aggregation of Perylene Diimide (PDI) and its derivatives strongly depends on the molecular structure, and therefore has great impact on the excited states. By regulating the molecular stacking such as monomer, dimer, J- and/or H-aggregate, the formation of different excited states is adjustable and controllable. In this study, we have synthesized two kinds of PDI derivatives - undecane-substituted PDI (PDI-1) and diisopropylphenyl-substituted PDI (PDI-2), and the films are fabricated with spin-coating method. By employing photoluminescence (PL), time-resolved photoluminescence (TRPL) and transient absorption (TA) spectroscopy, the excited-state dynamics of two PDI amorphous films have been investigated systematically. The result reveals that both films have formed excimer after photoexcitation mainly due to the stronger electronic coupling among molecule aggregate in the amorphous film. It should be noted that the excited state dynamics in PDI-2 shows a singlet fission like process, which is evidenced by the appearance of triplet state absorption. This study provides the dynamics of excited state in amorphous PDI films, and pave the way for better understanding and adjusting the excited state of amorphous films.
\end{abstract}

Keywords: PDI derivatives; singlet state; excimer; triplet state; molecule stacking; transient absorption 


\section{Introduction}

Perlyene Diimides (PDI) were employed as dye or pigments initially, and had been extensively used in industrial. ${ }^{1}$ Due to its wide absorption of visible-light and high stability, many studies have focused on its potential applications in photocatalysts ${ }^{2-5}$, anticancer treatment, ${ }^{3,6}$ and photovoltaic etc. ${ }^{7-9}$ The flat molecular structure in PDI facilitates intermolecular interactions with neighboring molecules, as a result, supermolecules can be formed by molecular stacking with side-by-side and/or head-to-tail in the solid state, in which strong electron coupling occurs among neighboring molecules. ${ }^{10-11}$ In these supermolecules, the stacking modes have great impact on their optical properties, catalytic performance, solubility, and so on. Moreover, substitution at imide nitrogen with various chromophores shows little effect on optical and electronic properties but can be used to tailor the aggregation and solubility of PDI molecules. ${ }^{1}$

The inter-chromosphere geometries have great impact on the behavior of PDIs. By synthesizing with various substitutions at imide nitrogen, the angle, $\pi-\pi$ interplanar distance can be controlled, in which the excited state can be adjusted dramatically. ${ }^{12-17}$ Wasielewski et al. shows that the broader scope of inter-chromophore geometry that lead to favorable electronic coupling and charge transfer (CT) states involved in singlet fission (SF). ${ }^{14}$ By controlling the structures, it is possible to increase the triplet rate that can improve the efficiency in PDI-based solar cells. By combing with the calculation, they demonstrated when the stacked molecules are rotated by $23^{\circ}$, the electronic coupling can result in a higher SF. Roberts et. al. found that CT-mediated coupling is related to the stacking, and the singlet fission is sensitive to the thin film structure. They reported that the triplet excitons are produced with $178 \%$ when the PDI molecules are arranged cofacially with a slip along their long axis of $\sim 3 \AA .{ }^{14}$ In addition, the aggregation behaves as an important factor for controlling the formation of excited state as well as the dynamics. By controlling the aggregation as dimer or oligomer, excited states dynamics varies with stacking manner significantly. ${ }^{18-20} \mathrm{Kim}$ et. al. reported the formation of excimer in both dimer and aggregate in PDIs, the formation time is faster in aggregate than that in dimer. ${ }^{18}$ The dimer also shows strong 
electronic coupling between two molecules that cause the formation of excimer.

The previous studies mainly focused on adjusting molecular aggregation in solution such as monomer and/or dimer as well as in solid state in status of polycrystalline. ${ }^{21-24}$ Few studies were carried out on the formation and dynamics of excited state in amorphous PDI film that can be fabricated with spin coating method. Compared with the film fabrication with vapor deposit method, the spin coating method has the advantages of low cost and easy to operate, which has been widely employed in large production. However, most films fabricated by spin coating are amorphous, and the aggregates are complex which include monomer, dimer, J- and/or H-aggregate, and even formation of polycrystalline phase partially. A study of understanding the excited states of amorphous films is indispensable, which help us build up the relationship between the formation and dynamics of excited state and the molecular structure in amorphous films. In this study, amorphous films of two PDI derivatives with different imide-substitutes and high soluble are fabricated by spin coating method. By carefully adjusting spin-speed and casting time, PDI films with good surface flatness and high uniformity are obtained. By combing with time-resolved photoluminescence (TRPL) and transient absorption (TA) spectroscopy, we have studied the excited state dynamics of the two PDI films with different imide-substituents. The experimental results demonstrate that both PDI films undergo complex aggregation during spin coating process: both $\mathrm{J}$ - and H-aggregate are observable from the steady absorption and PL spectra. On the other hand, the difference between the two films is also obvious: the undecane-substituted PDI film shows more closely cofacial stacks, in which the H-aggregate plays more important role in the optical properties. In contrast, diisopropylphenyl-substituted PDI film shows weak interaction among PDI molecules, in which the head-to-tail alignment is preferred during the film growth, as a result, the molecules are stacked with more J-aggregate like. Femtosecond TA spectra reveal that both films show pronounced ground state bleach (GSB) with recovery time longer than $8 \mathrm{~ns}$, and relaxation of excited state absorption (ESA) shows a typical lifetime of $\sim 1$ ns. Global fittings demonstrate that the intermediate states are different, in which the PDI-1 experiences a symmetry breaking before forming an excimer-like state and 
decaying to the ground state for several ns. On the other hand, the intermediate state in PDI-2 can be attributed to the formation of excimer and subsequently trapped by triplet state via a singlet fission. Our study demonstrates that after photoexcitation in amorphous PDI films, the excimer is formed in both films, which plays dominant role in PDI-1 film with more H-aggregate. By contrast in PDI-2 film with more J-aggregate, triplet state competes with the formation of excimer effectively. More importantly, we infer that the singlet fission may also occur even in amorphous films.

\section{Experimental section}

Synthesis of two PDI derivatives: Perylene-3,4,9,10 tetracarboxylic dianhydride (PTCDA), 2,6-diisopropylaniline, and imidazole were purchased from Energy Chemical and used as received. Undecan-6-amine was synthesized according to the literature. ${ }^{25}$ Analytical thin-layer chromatography (TLC) was performed on silica gel $60 \mathrm{~F}_{254}$ glass plates. The silica gel $60 \mathrm{H}$ (200-300 mesh) manufactured by Qingdao Haiyang Chemical Group Co. (China) was used for general chromatography. NMR spectra were recorded on Bruker AV 400 spectrometer at $400 \mathrm{MHz}\left({ }^{1} \mathrm{H}\right.$ NMR), 101 $\mathrm{MHz}\left({ }^{13} \mathrm{C}\right.$ NMR). The residual solvent signals were used as references for ${ }^{1} \mathrm{H}$ and ${ }^{13} \mathrm{C}$ NMR spectra and the chemical shifts were converted to the TMS scale $\left(\mathrm{CDCl}_{3}: \delta \mathrm{H}=\right.$ $7.26 \mathrm{ppm}, \delta \mathrm{C}=77.16 \mathrm{ppm})$. The detailed synthesis for undecane-substituted PDI (PDI-1) and diisopropylphenyl-substituted PDI (PDI-2) are provided in the Supplementary Material (SM). The scheme 1 presents the chemical structures of the two PDI derivatives.

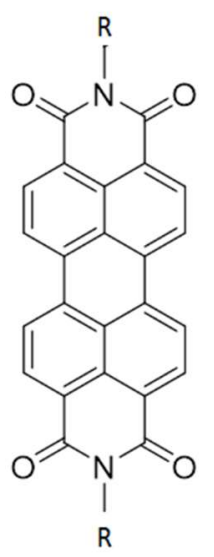

$$
\mathrm{R}_{1}=\mathrm{C}_{5} \mathrm{H}_{11} \mathrm{C}_{5} \mathrm{H}_{11}
$$

PDI-1 (undecane-substituted PDI): 2,9-di(undecane-6yl)anthra[2,1,9-def:6,5,10-d'e'f']diisoquinoline$1,3,8,10(2 \mathrm{H}, 9 \mathrm{H})$-tetraone

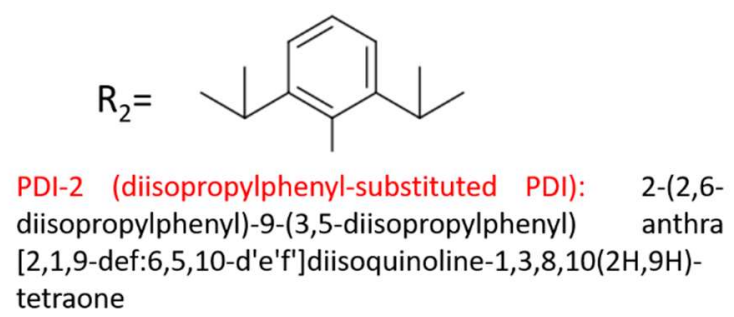

Scheme 1. The chemical structures of two PDI derivatives 
Thin Film Preparation: The two PDI films were obtained by spin-coating of corresponding PDI chloroform solution with concentration $15 \mathrm{mg} / \mathrm{ml}$ on $1-\mathrm{mm}$ thick fused silica substrate. The speed of rotation is adjusted to be $600-700 \mathrm{rpm}$ for $60 \mathrm{~s}$. The average thickness of the final film is determined to be about $300 \mathrm{~nm}$. X-ray diffraction (XRD) patterns of the films are shown in Figure S3 in SM, obviously the XRD patterns of both PDI-1 and PDI-2 films are almost same as that of the substrate, the fused silica, which demonstrate that the PDI spin-coating films are in amorphous phase.

Absorption and Fluorescence characterization: UV-visible absorption spectra of two PDI solution and films were carried out on Shimadzu spectrometer (UV-3600). Steady and transient photoluminescence (PL) spectra for both solution and films were carried out on Edinburgh $\alpha-1000$. A picosecond time-correlated single photon counting (TCSPC) system was used for time-resolved photoluminescence (TRPL) decay measurements. The samples were excited by a semiconductor laser (EPL-485 Edinburgh) with pulse width of $95 \mathrm{ps}$ and wavelength of $485 \mathrm{~nm}$. The instrumental response function (IRF) is better than $100 \mathrm{ps.}$

Transient absorption spectroscopy: The ultrafast spectroscopy of the two PDI films was carried out on transient absorption spectroscopy (ultrafast system, Hellos Fire Transient absorption spectrometer). Briefly, the light source was output from a commercial mode-locked Ti: sapphire laser (Coherent, Astrella tunable USP) with a central wavelength of $800 \mathrm{~nm}$, repetition rate of $1 \mathrm{kHz}$, and pulse duration of $35 \mathrm{fs}$, the incident pulse trains split into pump and probe pulses. The pump pulse was guided into an optical parametric amplifier system, which was used to tune the wavelength of the pump pulse in order to deliver the experimental-need wavelength beam, the pulse duration of the output from OPA was about 60 fs. The other $800 \mathrm{~nm}$-beam was focused on a $1 \mathrm{~mm}$-thick sapphire in order to produce a white light continuum with pulse duration of $120 \mathrm{fs}$, which acts as probe beam in the ultrafast system. An achromatic lens was used to focus the white continuum (probe) on the spot of $0.5 \mathrm{~mm}$ in diameter, which is rough half in size of the pump beam. An optical filter was used to remove the redundant $800 \mathrm{~nm}$-beam to produce the white light ranged from $450 \mathrm{~nm}$ 
to $780 \mathrm{~nm}$. The pump and probe beams were focused on the surface of sample overlappingly. The pump induced the transmission change of probe beam was collected by a high sensitive charge-coupled device. In this study the pump wavelength is fixed at $485 \mathrm{~nm}$ with fluence of about $3.9 \mu \mathrm{J} / \mathrm{cm}^{2}$, which is 10 times larger than that of the probe pulse. The pump induced absorption changes of probe beam $(\Delta \mathrm{A})$ was used to describe the optical induced change of the PDIs films from the transient absorption spectra. $\Delta \mathrm{A}<0 \quad(>0)$ indicates a photo-induced bleaching (absorption). All measurements were performed at room temperature.

\section{Experimental results}

\subsection{Steady State Absorption and Photoluminescence Study}

Figure 1 shows the intensity normalized steady absorption and PL spectra of the two PDIs in solution and film. The absorption and PL spectra of both PDIs in $10^{-5} \mathrm{M}$ chloroform solution show exact the same, in which absorption bands are located around 526,489 , and $458 \mathrm{~nm}$ for $0-0,0-1$, and $0-2$ vibronic bands, respectively. ${ }^{26}$ While the PL spectrum shows clear mirror symmetry with the absorption band, in which the $0-0,0-1$, and $0-2$ vibronic emission bands are located around 533, 573 and $620 \mathrm{~nm}$, respectively. As mentioned above, the imide-substituent of PDI in dilute solution has negligible effect on optical properties. Through the steady absorption, it draws a conclusion that PDIs demonstrate monomer form in low concentration solution. It is clear that the absorption and PL features in solution and films vary obviously. Comparing with the PDI maximum absorption in solution, the PDI-1 film shows blue shift of $\sim 27 \mathrm{~nm}$, indicating the dominant of $\mathrm{H}$-aggregate in film. On the other hand, the film absorption shows a flat and broad absorption band, and the onsite absorption has extended from $550 \mathrm{~nm}$ in solution to $700 \mathrm{~nm}$ in film. The PL spectrum shows the similar result as well: a broader and featureless emission band with large red shift $(142 \mathrm{~nm})$ relative to that of solution, which indicates J-aggregate has also existed in the film. The complex optical spectra demonstrate that aggregation in PDI-1 film can't be described with either J-aggregate or H-aggregate only. ${ }^{27}$ Additionally, we can also infer that molecules in PDI-1 form closely co-facial stacking, in which $\mathrm{H}$-aggregate plays dominated role accompanied with formation of 
J-aggregate during the film fabrication with spin-coating method. As illustrated in Fig. 1(b), the absorption and PL spectra of PDI-2 film show distinct features comparing with PDI-1. The PDI-2 film presents clear 0-0, 0-1 and 0-2 vibronic absorption bands, located around 535, 496 and $466 \mathrm{~nm}$, respectively, which resembles to that of the solution except for $\sim 10 \mathrm{~nm}$ red shifts. It is also noted that the PL spectrum of PDI-2 film also shows large red shift $(\sim 107 \mathrm{~nm})$ with respect to the solution. The most significant difference in PL spectra between PDI-2 and PDI-1 is that the PDI-2 film shows vibronic modulated emission resembling to the corresponding solution. Therefore we infer that the PDI-2 film undergoes head-to-tail stacking and the coupling between molecule nearby is weaker than PDI-1. From the PL spectra in Fig. 1 (a) and (b), both fluorescence spectra are broadened and red-shifted compared with the monomer counterpart, this pronounced feature is a typical character of PDI excimer formation, suggests that a charge transfer may emerge in both films. ${ }^{13}$
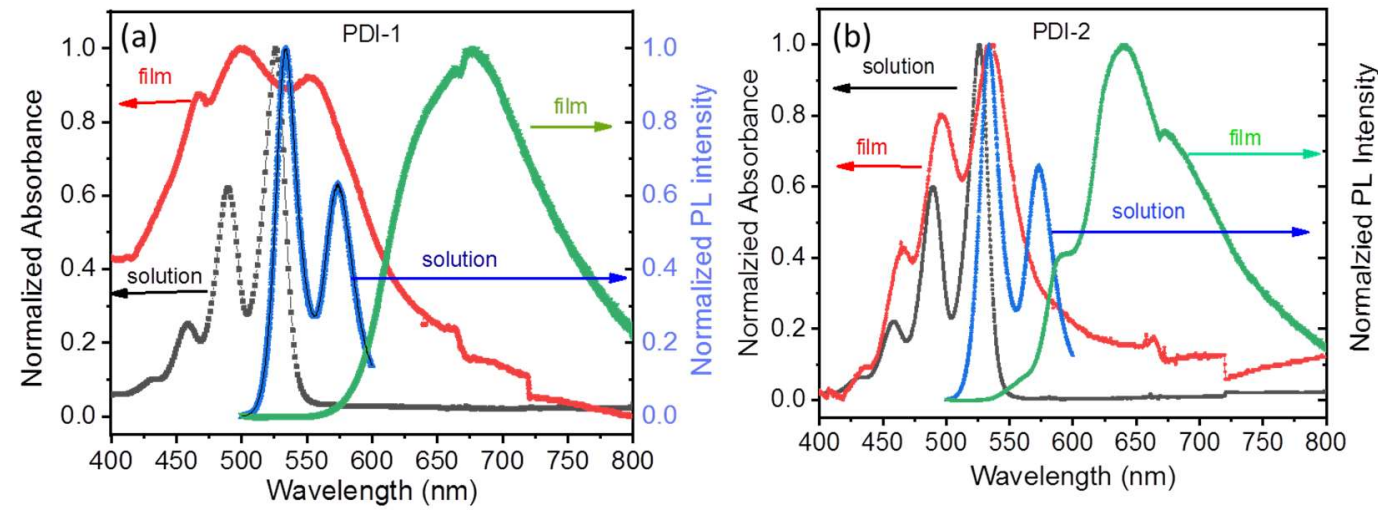

Figure 1. The intensity normalized UV-visible absorption and photoluminescence spectra of PDI-1 (a) and DPI-2 (b) in dilute $\mathrm{CHCl}_{3}$ solution and films, respectively.

\subsection{Time-Resolved Fluorescence Study}

Figure 2 presents the time-resolved PL of the two PDIs in solution and films, it is clear that the solution shows monoexponential decay with typical lifetime of $\sim 3.9 \mathrm{~ns}$. In contrast, the films show multiple relaxations, the fast relaxation time with typical lifetime of $\sim 1.0 \mathrm{~ns}$, which is 4-fold faster than that of PDI monomer in solution, indicating the presence of competing nonradiative decay processes in solid state. ${ }^{11}$ 
The reduced fluorescence lifetime and intensity can be attribute to the close packing that enhances electronic coupling and quench the fluorescence between molecules in films. In addition, each film displays a small, long-lived emission component that persists for $14.8 \mathrm{~ns}$ for PDI-1 and $7.0 \mathrm{~ns}$ for PDI-2, suggesting this emission originates from a process other than PDI monomer relaxation, and the decay from singlet state that will be discussed in the following. Figures 3(b) and (c) present transient fluorescence spectra collected at several selected delay times for PDI-1 (a) and PDI-2 (b), respectively. The 3D plots of transient PL are given in Fig. S4 PDI-1 and Fig. S5 for PDI-2 in SM. It clearly indicates that not a longer decay time, broader fluorescence feature but a more pronounced red-shift is observed with time going in the amorphous films comparing with the monomer counterpart in solution, which given that the excimer could be formed in both films. ${ }^{18}$

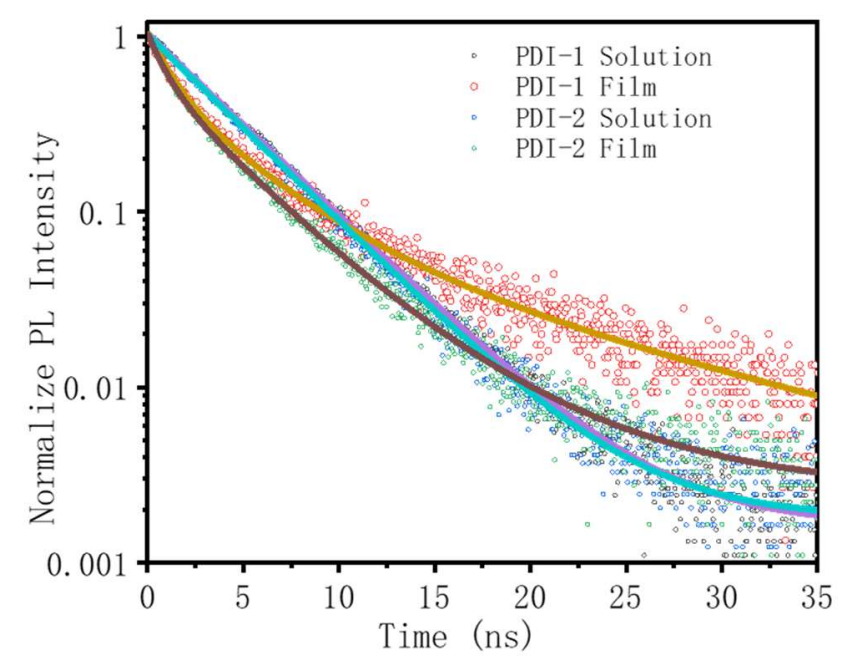

Figure 2. The intensity normalized PL spectra of PDI-1 and PDI-2 in dilute chloroform solution and films, respectively. 

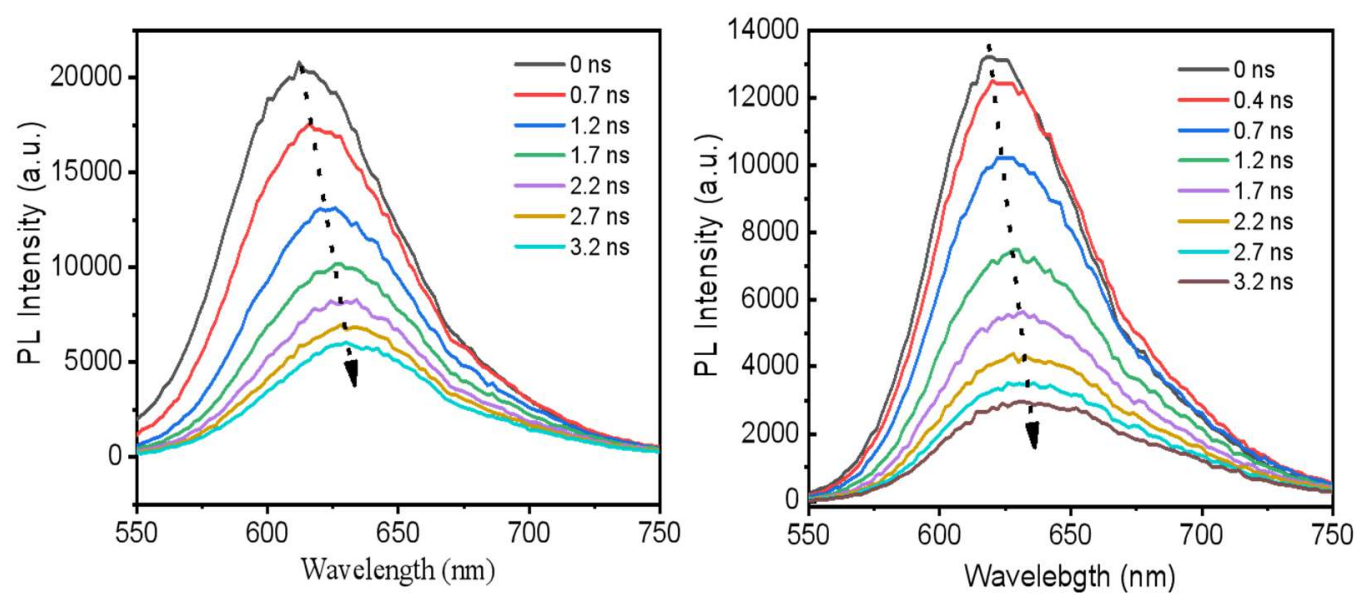

Figure 3. Transient fluorescence spectra collected at several selected delay times for PDI-1 (a) and PDI-2 (b) films. The arrows in the figure indicate the PL spectra shift with time.

\subsection{Transient Absorption Study}

In order to understand the side-chain influence on the excited state dynamics, we carried out transient absorption measurement on the two films. Figure 4(a) presents the TA spectra of PDI-1 film in visible region collected at several delay times after photoexcitation at $485 \mathrm{~nm}$. The GSB around $558 \mathrm{~nm}$ is clearly observed that responds to the maximum absorbance, i.e. 0-0 vibronic absorption as illustrated in Fig.1(a). In addition, two obvious photoinduced absorption peaks appear in $625 \mathrm{~nm}$ and $765 \mathrm{~nm}$, which come from the excited state absorption (ESA). The 3D plot of transient absorption is exhibited in Fig. S6 in SM. Focus on the photoabsorption signals at 625 $\mathrm{nm}$ and $765 \mathrm{~nm}$, both signals vanish completely when delay time is longer than $\Delta \mathrm{t}=1$ ns. However the photobelaching signal remains $\sim 10 \%$ of weight after $\Delta \mathrm{t}=7.5 \mathrm{~ns}$, which reveals the recovery of GSB is in the time scale much longer than the lifetime of singlet excited state. In order to learn more dynamical information of the photoexcitation, four featured wavelengths of 535, 558, 625 and $765 \mathrm{~nm}$ are selected to monitor the excited state dynamics after photoexcitation of $485 \mathrm{~nm}$. Figure 4(b) and 4(c) present the dynamical relaxations at each selected wavelength in a short time window (b) and a long time window (c), respectively. It can be seen from Fig. 4(c) that the recovery processes of GSB at both $535 \mathrm{~nm}$ and $558 \mathrm{~nm}$ do not reach baseline 
up to $7.5 \mathrm{~ns}$, the limit of our experimental setup. In contrast, the relaxation at $625 \mathrm{~nm}$ and $765 \mathrm{~nm}$ return to the initial sate within $1 \mathrm{~ns}$. This strongly suggests that an intermediate state can be assigned to trap singlet exciton, while the ground state remains unoccupied. What's more, the decaying features at $625 \mathrm{~nm}$ and $765 \mathrm{~nm}$ are different, which indicates the signals of some spices may overlap in ESA. Both decay profiles of $625 \mathrm{~nm}$ and $765 \mathrm{~nm}$ can be fitted with triple-exponential function, but the decay time in $765 \mathrm{~nm}$ is longer than that in $625 \mathrm{~nm}$, which suggest the different paths may exist for relaxation of 625 and $765 \mathrm{~nm}$. Through the Fig. 4(c), it can adapt that the sign of $\Delta \mathrm{A}$ at $625 \mathrm{~nm}$ changes from positive to negative around $\sim 1.9 \mathrm{~ns}$, which suggest the formation of excimer. Since the lifetime of fluorescence from singlet is about $1 \mathrm{~ns}$, the negative signal with lifetime over $8 \mathrm{~ns}$ can be attribute to the excimer, which has a life time of 14 ns according to TRPL spectra given in Fig. 2. Furthermore, the relaxation at $765 \mathrm{~nm}$ with recovery time of $1 \mathrm{~ns}$ is attribute to the relaxation of the excited state.
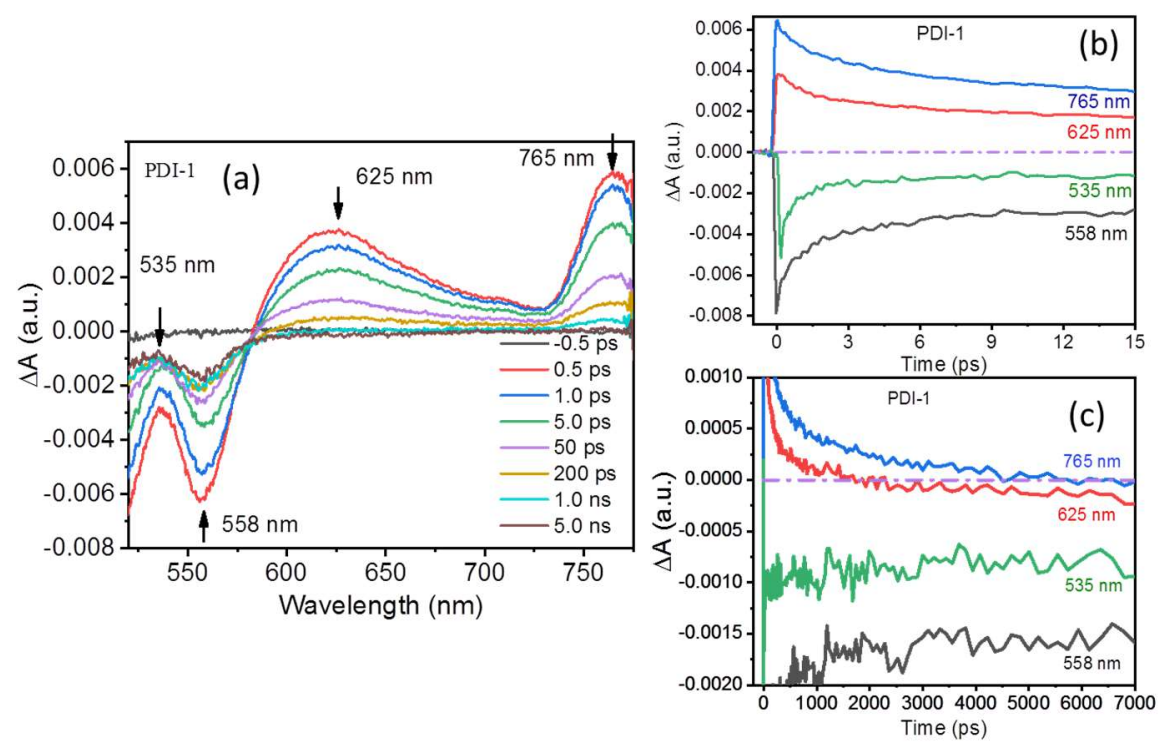

Figure 4. Transient absorption spectra of PDI-1 film after photoexcitation of $485 \mathrm{~nm}$. (a) TA spectra collected at delay times of $-0.5,0.5,1.0,5.0,50,200,1000,5000 \mathrm{ps}$, respectively. (b) Transient dynamics of PDI-1 at four selected wavelengths in short time window, and (c) Transient dynamics of PDI-1 at four selected wavelengths in long time window. 

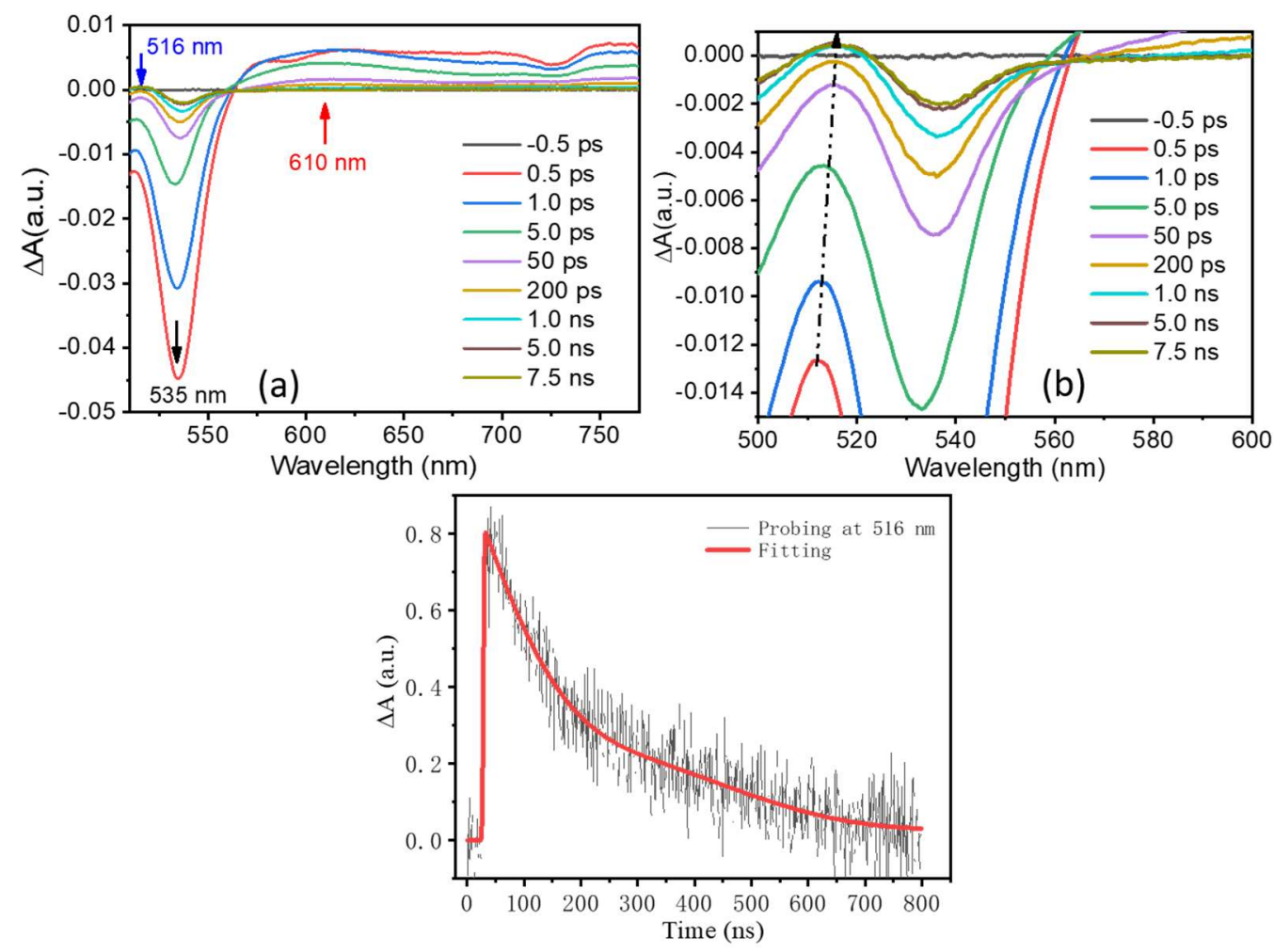

Figure 5. Transient absorption spectra of PDI-2 film after photoexcitation of $485 \mathrm{~nm}$. (a) TA spectra collected at delay times of $-0.5,0.5,1.0,5.0,50,200,1000,5000$, and $7500 \mathrm{ps,}$ respectively. (b) The enlargement of (c) TA spectra collected at $516 \mathrm{~nm}$ in long time scale $(\sim 1 \mu \mathrm{s})$ for PDI-2 film

Figure 5 (a) shows the TA spectra of PDI-2 at several selected delay times. Figure S7 in SM presents the 3D plots of the TA spectra. An obvious GSB is located at 535nm but the photoinduced signal $(600-750 \mathrm{~nm})$ is broad and flat compared with PDI-1. The broad featureless characterization indicates the formation of excimer that accord to the time resolved fluorescence. ${ }^{10,13,15}$ Interestingly, it notes that there is a weak signal centered around $516 \mathrm{~nm}$, in which the signal changes from negative in early delay time to positive in later delay time. It is more clear that the signal shows red shift and becomes broad with delay time as illustrated in Fig. 5(b), which reveals the emergence of a new specie. Figure 5 (c) shows the TA spectra around $516 \mathrm{~nm}$ in a long-time scanning $(\sim 1 \mu \mathrm{s})$, the positive signal exists for about $500 \mathrm{~ns}$, from which we can conclude that the signal at $516 \mathrm{~nm}$ changed from negative to positive with the lifetime of hundreds of ns can be attribute to the absorption of triplet. ${ }^{14,17}$ By referring 
to previous studies of PDI polycrystalline film, ${ }^{10,14}$ we infer that the formation of triplet could be caused by singlet fission process in our amorphous film, which competes with the formation of excimer after photoexcitation. However, the triplet state does not present in PDI-1 amorphous film, which demonstrates that formation of triplet strongly depends on the molecule stacking. It is well known that side-chain substitutions play dominate role in the molecule stacking during spin casting, therefore it is possible to monitor the formation of triplet by attaching different groups on the main frame of PDI molecule.

\subsection{Global Fitting and Photocarrier Dynamics}

According to the TA spectra of the two PDI films, we have demonstrated that the PDI-1 film has formed an excimer-like state, while the PDI-2 is featured by the triplet absorption after photoexcitation. However, many signals could be concealed due to the overlapping of different spectral components. ${ }^{28-30}$ In order to analyze the intermediate state of PDIs, SVD is performed, and the subsequently generated kinetic amplitude vectors are globally fitted..$^{31}$ Global fitting analysis can reduce the data to a superposition of time independent spectra, which can help us to obtain the main characters and some minor species that are hard to separate in common TA spectra. Commercial software, Surface Xplorer, is used to perform the SVD and global fitting analysis.
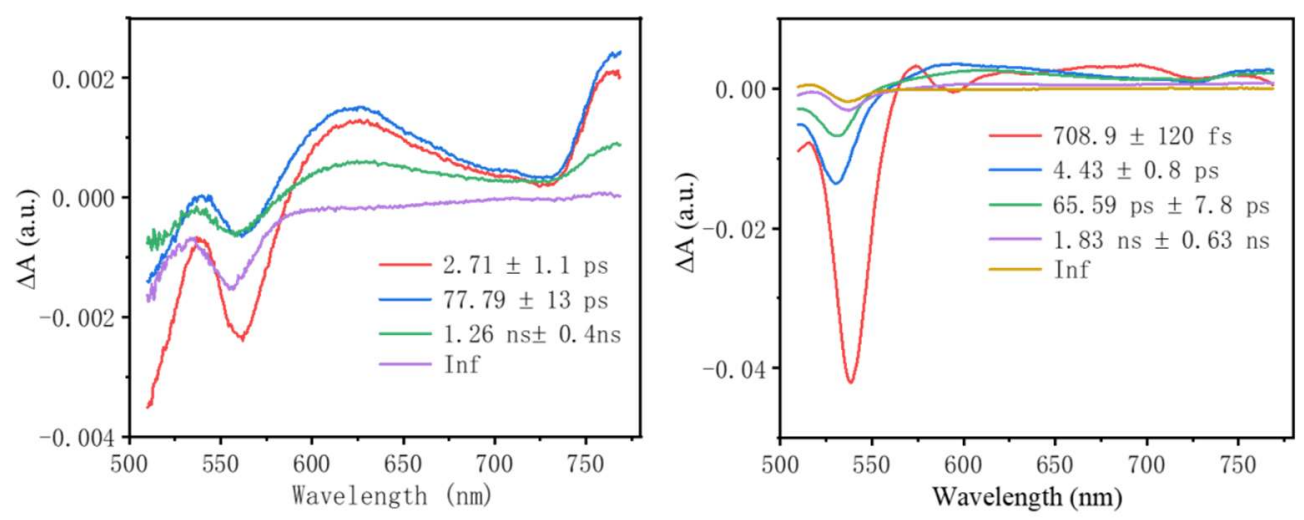

Figure 6. Decay-associated difference spectra for PDI-1 (a) and PDI-2 (b). 
In all cases the data can be best fitted with a four components in PDI-1 and five components in PDI-2 as shown in Fig. 6, in which the corresponding Decay-Associate Differential Spectra (DADS) are exhibited as well. The first one in PDI-1 and the first two in PDI-2 time constants $\left(<5\right.$ ps) can attribute to vibrational cooling. ${ }^{29}$ The time constant about hundreds of ps to $\sim 1$ ns time scale can be ascribed to decay of excited state accompanying with change of both spectral shape and proportion. Interestingly, the absorption weight for the component of $77.79 \mathrm{ps}$ is higher than that of $2.71 \mathrm{ps}$, which indicates the formation of a new specie. Considering the identical spectral shape of the two components, a symmetry breaking is inferred to take place after photoexcitation that causes electron separation. As a result, the phenomena mentioned above can be ascribed to the anion or cation absorption. ${ }^{13}$ The time constant of $1.26 \mathrm{~ns}$ accords to the singlet emission, while the component of inf depicts the emission of excmier. ${ }^{13,15}$ The dynamics in PDI-2 varies differently. The first two time constants ( $<5 \mathrm{ps}$ ) can be ascribed to vibrational cooling as well. The time constant of $\sim 65.59 \mathrm{ps}$ with broad flat feature indicates the formation of excimer, ${ }^{15}$ and the component of $\sim 1.83 \mathrm{~ns}$ is according to the singlet emission. More importantly, the time constant of inf can be attribute to the triplet absorption since the signal at $516 \mathrm{~nm}$ remains positive up to $500 \mathrm{~ns}$, the excited state absorption from 525 to $750 \mathrm{~nm}$ has been back to the baseline within $1 \mathrm{~ns} .{ }^{14-15,18}$ In brief, the results from TA spectra combined with Global fitting reveal that the PDI-1 film has experienced symmetry breaking before the generation of excimer, and then return to the ground state via emission. By contrast, the excited state relaxation of PDI-2 film is dominated by the long-lived triplet states by competing with excimer state, and the singlet fission is possible process for the formation of triplet states.

\section{Conclusions}

In conclusion, two PDI films, i.e. undecane- and diisopropylphenyl-substitutent of PDI at imide position, are fabricated with spin coating technique. Two amorphous films show complex absorption and PL spectra due to the complex short- and long-range molecular interactions. In general, the undecane-substituted PDI film 
shows more H-aggregate like characteristic, while the diisopropylphenyl-substituted PDI film has more J-aggregate like stacking. The electron coupling in PDI-1 is stronger than that in PDI-2 according to different absorption features. The TA spectra of the two films reveal that the excited-state dynamics of two films are dominated by different intermediate states. For PDI-1 film, photoexcitation leads to the formation of symmetry breaking before forming excimer that dominates the dynamics of excited states. In contrast, the excited state dynamics in PDI-2 is dominated by triplet state that competes with formation of excimer. The time resolved photoluminescence spectra show good agreement with the TA measurement. Our study shows the excited state in amorphous films are quite complex. The appearance of long-lived triplet in PDI-2 indicates the singlet fission may also occur in amorphous PDI film. What's more, the PDIs also show strong coupling as the excimer exist in both films. Our work paves the way for the study of amorphous films and shows interesting photophysical phenomenon. More importantly, the study sheds light for better understanding the dynamics of excited state for amorphous films fabricated with spin-coating technique.

\section{Declaration of Competing Interest}

The authors declare that they have no known competing financial interests or personal relationships that could have appeared to influence the work reported in this paper.

\section{CRediT authorship contribution statement}

Qiushi Ma: Conceptualization, Investigation, Formal Analysis, Writing - review \& editing. Cheng-Wei Ju: Conceptualization, Investigation. Ruihua Pu: Investigation. Wenjie Zhang: Investigation. Xian Lin: Investigation. Yihan Chen: Conceptualization, Resources. Weimin Liu: Conceptualization, Resources.

\section{Acknowledgments}

We are grateful to Dr. Dongbing Zhao and Dr. Liping Lv for their helpful discussion. This work was supported by the National Natural Science Foundation of China (NSFC, No. 11774233). 


\section{References}

1. Huang, C.; Barlow, S.; Marder, S. R., Perylene-3,4,9,10-Tetracarboxylic Acid Diimides: Synthesis, Physical Properties, and Use in Organic Electronics. J Org Chem 2011, 76, 2386-407.

2. Gao, Q.; Xu, J.; Wang, Z.; Zhu, Y., Enhanced Visible Photocatalytic Oxidation Activity of Perylene Diimide/G-C3n4 N-N Heterojunction Via ח-ח Interaction and Interfacial Charge Separation. Applied Catalysis B: Environmental 2020, 271.

3. Liu, D.; Wang, J.; Bai, X.; Zong, R.; Zhu, Y., Self-Assembled Pdinh Supramolecular System for Photocatalysis under Visible Light. Adv Mater 2016, 28, 7284-90.

4. Zhang, Z.; Chen, X.; Zhang, H.; Liu, W.; Zhu, W.; Zhu, Y., A Highly Crystalline Perylene Imide Polymer with the Robust Built-in Electric Field for Efficient Photocatalytic Water Oxidation. Adv Mater 2020, 32, e1907746.

5. Zhang, Q.; Jiang, L.; Wang, J.; Zhu, Y.; Pu, Y.; Dai, W., Photocatalytic Degradation of Tetracycline Antibiotics Using Three-Dimensional Network Structure Perylene Diimide Supramolecular Organic Photocatalyst under Visible-Light Irradiation. Applied Catalysis B: Environmental 2020, 277.

6. Wang, J.; Liu, D.; Zhu, Y.; Zhou, S.; Guan, S., Supramolecular Packing Dominant Photocatalytic Oxidation and Anticancer Performance of Pdi. Applied Catalysis B: Environmental 2018, 231, 251-261.

7. Nattestad, A.; Mozer, A. J.; Fischer, M. K.; Cheng, Y. B.; Mishra, A.; Bauerle, P.; Bach, U., Highly Efficient Photocathodes for Dye-Sensitized Tandem Solar Cells. Nat Mater 2010, 9, 31-5.

8. Hu, H., et al., Effect of Ring-Fusion on Miscibility and Domain Purity: Key Factors Determining the Performance of Pdi-Based Nonfullerene Organic Solar Cells. Advanced Energy Materials 2018, 8.

9. Tamai, Y.; Fan, Y.; Kim, V. O.; Ziabrev, K.; Rao, A.; Barlow, S.; Marder, S. R.; Friend, R. H.; Menke, S. M., Ultrafast Long-Range Charge Separation in Nonfullerene Organic Solar Cells. ACS Nano 2017, 11, 12473-12481.

10. Young, R. M.; Wasielewski, M. R., Mixed Electronic States in Molecular Dimers: Connecting Singlet Fission, Excimer Formation, and Symmetry-Breaking Charge Transfer. Acc Chem Res 2020, 53, 1957-1968.

11. Naqvi, S.; Kumar, M.; Kumar, R., Facile Synthesis and Evaluation of Electron Transport and Photophysical Properties of Photoluminescent Pdi Derivatives. ACS Omega 2019, 4, 19735-19745.

12. Roberts, S. T., Energy Transport: Singlet to Triplet and Back Again. Nat Chem 2015, 7, 764-5.

13. Ramirez, C. E.; Chen, S.; Powers-Riggs, N. E.; Schlesinger, I.; Young, R. M.; Wasielewski, M. R., Symmetry-Breaking Charge Separation in the Solid State: Tetra(Phenoxy)Perylenediimide Polycrystalline Films. J Am Chem Soc 2020, 142, 18243-18250.

14. Le, A. K.; Bender, J. A.; Arias, D. H.; Cotton, D. E.; Johnson, J. C.; Roberts, S. T., Singlet Fission Involves an Interplay between Energetic Driving Force and Electronic Coupling in Perylenediimide Films. J Am Chem Soc 2018, 140, 814-826.

15. Margulies, E. A.; Logsdon, J. L.; Miller, C. E.; Ma, L.; Simonoff, E.; Young, R. M.; Schatz, G. C.; Wasielewski, M. R., Direct Observation of a Charge-Transfer State Preceding High-Yield Singlet Fission in Terrylenediimide Thin Films. J Am Chem Soc 2017, 139, 663-671.

16. Margulies, E. A.; Miller, C. E.; Wu, Y.; Ma, L.; Schatz, G. C.; Young, R. M.; Wasielewski, M. R., Enabling Singlet Fission by Controlling Intramolecular Charge Transfer in Pi-Stacked Covalent Terrylenediimide Dimers. Nat Chem 2016, 8, 1120-1125.

17. Masoomi-Godarzi, S.; Hall, C. R.; Zhang, B.; Gregory, M. A.; White, J. M.; Wong, W. W. H.; Ghiggino, K. P.; Smith, T. A.; Jones, D. J., Competitive Triplet Formation and Recombination in Crystalline Films of Perylenediimide Derivatives: Implications for Singlet Fission. The Journal of 
Physical Chemistry C 2020, 124, 11574-11585.

18. Son, M.; Park, K. H.; Shao, C.; Würthner, F.; Kim, D., Spectroscopic Demonstration of Exciton Dynamics and Excimer Formation in a Sterically Controlled Perylene Bisimide Dimer Aggregate. The Journal of Physical Chemistry Letters 2014, 5, 3601-3607.

19. Schubert, A.; Settels, V.; Liu, W.; Wurthner, F.; Meier, C.; Fink, R. F.; Schindlbeck, S.; Lochbrunner, S.; Engels, B.; Engel, V., Ultrafast Exciton Self-Trapping Upon Geometry Deformation in Perylene-Based Molecular Aggregates. J Phys Chem Lett 2013, 4, 792-6.

20. Long, S.; Wang, Y.; Vdovic, S.; Zhou, M.; Yan, L.; Niu, Y.; Guo, Q.; Xia, A., Energy Transfer and Spectroscopic Characterization of a Perylenetetracarboxylic Diimide (Pdi) Hexamer. Phys Chem Chem Phys 2015, 17, 18567-76.

21. Myong, M. S.; Zhou, J.; Young, R. M.; Wasielewski, M. R., Charge-Transfer Character in Excimers of Perylenediimides Self-Assembled on Anodic Aluminum Oxide Membrane Walls. The Journal of Physical Chemistry C 2020, 124, 4369-4377.

22. Brown, K. E.; Salamant, W. A.; Shoer, L. E.; Young, R. M.; Wasielewski, M. R., Direct Observation of Ultrafast Excimer Formation in Covalent Perylenediimide Dimers Using near-Infrared Transient Absorption Spectroscopy. J Phys Chem Lett 2014, 5, 2588-93.

23. Cook, R. E.; Phelan, B. T.; Kamire, R. J.; Majewski, M. B.; Young, R. M.; Wasielewski, M. R., Excimer Formation and Symmetry-Breaking Charge Transfer in Cofacial Perylene Dimers. J Phys Chem A 2017, $121,1607-1615$.

24. Kaufmann, C.; Kim, W.; Nowak-Krol, A.; Hong, Y.; Kim, D.; Wurthner, F., Ultrafast Exciton Delocalization, Localization, and Excimer Formation Dynamics in a Highly Defined Perylene Bisimide Quadruple Pi-Stack. J Am Chem Soc 2018, 140, 4253-4258.

25. Yano, Y.; Wang, F.; Mitoma, N.; Miyauchi, Y.; Ito, H.; Itami, K., Step-Growth Annulative Pi-Extension Polymerization for Synthesis of Cove-Type Graphene Nanoribbons. J Am Chem Soc 2020, 142, 1686-1691.

26. Wang, X.; Zeng, T.; Nourrein, M.; Lai, B.-H.; Shen, K.; Wang, C.-L.; Sun, B.; Zhu, M., Concentration-Dependent Self-Assembly Structures of an Amphiphilic Perylene Diimide with Tri(Ethylene Glycol) Substituents at Bay Positions. RSC Advances 2017, 7, 26074-26081.

27. Oleson, A., et al., Perylene Diimide-Based Hj- and Hj-Aggregates: The Prospect of Exciton Band Shape Engineering in Organic Materials. The Journal of Physical Chemistry C 2019, 123, 20567-20578.

28. Qin, C.; Liu, H.; Sun, S.; Zhou, Z.; Liu, Y., Ultrafast Investigation of Intramolecular Proton Transfer Dynamics and Vibration Relaxation in 1,8-Dihydroxyanthraquinone. Journal of Molecular Structure 2020.

29. Dozova, N.; Ventelon, L.; Clermont, G.; Blanchard-Desce, M.; Plaza, P., Excited-State Symmetry Breaking of Linear Quadrupolar Chromophores: A Transient Absorption Study. Chemical Physics Letters 2016, 664, 56-62.

30. Kakitani, Y.; Hou, A.; Miyasako, Y.; Koyama, Y.; Nagae, H., Rates of the Initial Two Steps of Electron Transfer in Reaction Centers from Rhodobacter Sphaeroides as Determined by Singular-Value Decomposition Followed by Global Fitting. Chemical Physics Letters 2010, 492, 142-149.

31. van Stokkum, I. H.; Larsen, D. S.; van Grondelle, R., Global and Target Analysis of Time-Resolved Spectra. Biochim Biophys Acta 2004, 1657, 82-104. 